\title{
Early enteral nutrition and total parenteral nutrition on the nutritional status and blood glucose in patients with gastric cancer complicated with diabetes mellitus after radical gastrectomy
}

\author{
JUNLI WANG $^{1}$, JIAMIN ZHAO ${ }^{2}$, YANLING ZHANG ${ }^{3}$ and CHONG LIU $^{4}$ \\ ${ }^{1}$ Department of Gastroenterology, The First People's Hospital of Jinan, Jinan, Shandong 250013; \\ ${ }^{2}$ Department of Urology, No. 89 Hospital of the People's Liberation Army, Weifang, Shandong 261000; \\ ${ }^{3}$ Department of Cardiovascular Medicine, The First People's Hospital of Jinan, Jinan, \\ Shandong 250013; ${ }^{4}$ Department of Gastrointestinal Surgery, The Affiliated Hospital of \\ Weifang Medical University, Weifang, Shandong 261041, P.R. China
}

Received October 10, 2017; Accepted May 9, 2018

DOI: $10.3892 /$ etm.2018.6168

\begin{abstract}
Effects of early enteral nutrition (EEN) or total parenteral nutrition (TPN) support on nutritional status and blood glucose in patients with gastric cancer complicated with diabetes mellitus after radical gastrectomy were investigated. One hundred and twenty-nine patients with gastric cancer complicated with diabetes mellitus type 2 admitted to the First People's Hospital of Jinan (Jinan, China), from June 2012 to June 2016 were selected into the study. According to different nutrition support pathways, these patients were randomly divided into the EEN group and the TPN group. The improvement of nutritional indexes, postoperative complications, gastrointestinal function recovery and perioperative blood glucose fluctuation were compared between the two groups. On the 4th day after operation, the improvement levels of total bilirubin (TBL), alanine aminotransferase (ALT), aspartate transaminase (AST), total protein (TP), prealbumin (PAB), hemoglobin (HGB) and weight (Wt) in the EEN group were significantly higher than those in the conventional group $(\mathrm{P}<0.05)$. There were no significant differences between the two groups on the 8th day after operation $(\mathrm{P}>0.05)$. No patients had complications in the EEN group, while a total of 29 patients in the TPN group suffered adverse reactions, indicating that the incidence rate of complications in the EEN group was significantly lower than that in the TPN group $(\mathrm{P}<0.05)$.
\end{abstract}

Correspondence to: Dr Chong Liu, Department of Gastrointestinal Surgery, The Affiliated Hospital of Weifang Medical University, 2428 Yuhe Road, Kui wen County, Weifang, Shandong 261041, P.R. China

E-mail: chong223322@163.com

Key words: gastric cancer, diabetes mellitus type 2, early enteral nutrition, total parenteral nutrition, blood glucose
The postoperative evacuation time was earlier, hospitalization time was shorter and cost of postoperative hospitalization was less in the EEN group than those in the TPN group, and the differences were statistically significant $(\mathrm{P}<0.05)$. The blood glucose fluctuation values at fasting and $2 \mathrm{~h}$ after a meal in the TPN group were higher than those in the EEN group within 8 days after operation, and the differences were statistically significant $\left(\chi^{2}=13.219, \mathrm{P}=0.002 ; \chi^{2}=20.527, \mathrm{P}<0.001\right)$. EEN support provides nutrition for patients with gastric cancer complicated with diabetes mellitus after radical gastrectomy, which is worthy of clinical promotion as it maintains good nutritional status, produces few postoperative complications and keeps the blood glucose level stable, by which the postoperative evacuation time is early, the hospitalization time is short and the cost is low.

\section{Introduction}

Gastric cancer has the highest incidence rate among malignant tumors in China, and this rate is increasing year by year, which is the leading threat to the health of people. At the same time, the incidence rate of diabetes mellitus is increased with the changes in lifestyles and the rising trend of the aging of population (1). Surgery-based comprehensive treatment is still the preferred effective treatment for patients with opportunistic gastric cancer complicated with diabetes mellitus. Most patients with gastric cancer complicated with diabetes mellitus have cellular immune dysfunction, malnutrition, poor cardiopulmonary function and decreased tissue repair capacity and other symptoms because of preoperative tumor consumption and intestinal preparation, postoperative fasting, and the surgical trauma stress keeps the body at high metabolic state, which further increases the patient's blood glucose level, thus leading to poor control of blood glucose and aggravating malnutrition (2). Surgical curative effect will be affected by postoperative hyperglycemia and malnutrition, and the incidence rate of postoperative complications 
Table I. General information of patients in the two groups.

\begin{tabular}{|c|c|c|c|c|}
\hline Characteristics & EEN group $(n=66)$ & TPN group $(n=63)$ & $\mathrm{t}$ value & P-value \\
\hline $\operatorname{Sex}(n)$ & & & 1.769 & 0.168 \\
\hline Male & 34 & 32 & & \\
\hline Female & 32 & 31 & & \\
\hline Age (years) & $48.07 \pm 7.45$ & $48.21 \pm 6.78$ & 2.126 & 0.073 \\
\hline Preoperative complications (n) & & & 1.912 & 0.089 \\
\hline Hypertension & 12 & 11 & & \\
\hline Coronary heart disease & 8 & 7 & & \\
\hline Peripheral neuropathy & 3 & 2 & & \\
\hline Tumor, node and metastasis (TNM) staging & & & 1.901 & 0.094 \\
\hline Stage I & 9 & 8 & & \\
\hline Stage II & 21 & 20 & & \\
\hline Stage III & 36 & 35 & & \\
\hline Histological grading & & & 2.313 & 0.065 \\
\hline G1 & 23 & 23 & & \\
\hline $\mathrm{G} 2$ & 29 & 28 & & \\
\hline G3 & 14 & 12 & & \\
\hline Operation methods (n) & & & 1.834 & 0.152 \\
\hline Billroth I operation & 10 & 9 & & \\
\hline Billroth II operation & 45 & 44 & & \\
\hline Total gastrectomy & 11 & 10 & & \\
\hline
\end{tabular}

Comparisons of general information between the two groups, $\mathrm{P}>0.05$.

is increased, thus affecting the body's functional status of patients (3). The nutrition support is an important means to promote the rehabilitation of patients, but how to better improve the postoperative nutritional status and control the blood glucose level of patients with gastric cancer complicated with diabetes mellitus is the focus and difficulty of the current research. Therefore, in this study, patients with gastric cancer complicated with diabetes mellitus were treated with early enteral nutrition (EEN) or total parenteral nutrition (TPN) support after radical gastrectomy, thus helping explore the effects of different nutrition supports on the nutritional status and blood glucose of patients, which is expected to provide a certain guidance for clinical operations.

\section{Materials and methods}

Clinical data. One hundred and twenty-nine patients with complete clinical data and pathologically confirmed as gastric cancer complicated with diabetes mellitus after operation who were admitted to the First People's Hospital of Jinan and received radical gastrectomy from June 2012 to June 2016 were selected into the study. According to the diagnostic criteria for diabetes mellitus type 2 issued by the American Diabetes Association (ADA) in 2006, the patients had diabetes mellitus type 2 (4). Inclusion criteria: Patients with primary gastric cancer and diabetes mellitus received no preoperative treatment and were complicated with no other malignancies. Exclusion criteria: Patients with severe liver or renal function impairment. According to different nutritional pathways, patients were randomly divided into the EEN group $(n=66)$ and the TPN group $(n=63)$. In the EEN group, there were 34 males and 32 females at the mean age of $48.07 \pm 7.45$ years. The preoperative complications included hypertension $(n=12)$, coronary heart disease $(n=8)$ and peripheral neuropathy $(n=3)$. Pathological features of patients: Stage I $(n=9)$, Stage II $(n=21)$ and Stage III $(n=36)$; histological grading: G1 $(n=23)$, G2 $(n=29)$ and G3 $(n=14)$; operation methods: Billroth I $(n=10)$, Billroth II $(n=45)$ and total gastrectomy $(n=11)$. In the TPN group, there were 32 males and 31 females at the mean age of $48.21 \pm 6.78$ years. Preoperative complications included hypertension $(n=11)$, coronary heart disease $(n=7)$ and peripheral neuropathy $(n=2)$. Pathological features of patients: Stage I $(n=8)$, Stage II $(n=20)$ and Stage III $(n=35)$; histological grading: G1 $(n=23), \mathrm{G} 2(\mathrm{n}=28)$ and G3 $(\mathrm{n}=12)$; operation methods: Billroth I operation $(n=9)$, Billroth II operation $(n=44)$ and total gastrectomy $(n=10)$. There were no significant differences between the two groups in general clinical data ( $P>0.05)$, and the baseline data were relatively consistent and comparable. This study was approved by the Ethics Committee of the First People's Hospital of Jinan, (Jinan, China). Patients were informed of the condition and signed informed consent. Preoperative preparation was actively conducted (Table I).

General clinical data records. General clinical data included sex, age, weight (patients were weighed in fasting state, wearing unlined dress and no hat or shoes), past medical 
Table II. Comparison of the improvement of nutritional indexes between the two groups of patients (mean \pm SD).

\begin{tabular}{|c|c|c|c|c|c|c|}
\hline \multirow[b]{2}{*}{ Index } & \multicolumn{3}{|c|}{ EEN group } & \multicolumn{3}{|c|}{ TPN group } \\
\hline & $\begin{array}{l}\text { Before } \\
\text { operation }\end{array}$ & $\begin{array}{l}\text { On the 4th day } \\
\text { after operation }\end{array}$ & $\begin{array}{l}\text { On the 8th day } \\
\text { after operation }\end{array}$ & $\begin{array}{l}\text { Before } \\
\text { operation }\end{array}$ & $\begin{array}{l}\text { On the 4th day } \\
\text { after operation }\end{array}$ & $\begin{array}{l}\text { On the 8th day } \\
\text { after operation }\end{array}$ \\
\hline TBL (U/mmol) & $10.65 \pm 3.63$ & $12.34 \pm 4.61^{\mathrm{b}}$ & $11.82 \pm 2.38$ & $11.07 \pm 3.46$ & $26.43 \pm 4.69$ & $10.56 \pm 3.72$ \\
\hline ALT (U/mmol) & $26.12 \pm 4.34$ & $29.05 \pm 4.39^{\mathrm{a}, \mathrm{b}}$ & $25.64 \pm 3.84$ & $25.78 \pm 4.26$ & $41.27 \pm 5.39^{\mathrm{a}}$ & $27.21 \pm 4.37$ \\
\hline $\operatorname{AST}(\mathrm{U} / \mathrm{mmol})$ & $26.57 \pm 3.67$ & $35.45 \pm 4.36^{\mathrm{a}, \mathrm{b}}$ & $26.64 \pm 4.52$ & $27.35 \pm 3.51$ & $54.81 \pm 5.45^{\mathrm{a}}$ & $28.62 \pm 4.33$ \\
\hline $\mathrm{TP}(\mathrm{g} / \mathrm{l})$ & $63.64 \pm 4.52$ & $59.09 \pm 5.64^{\mathrm{a}, \mathrm{b}}$ & $65.48 \pm 4.65$ & $63.84 \pm 4.58$ & $43.26 \pm 4.07^{\mathrm{a}}$ & $64.21 \pm 5.18$ \\
\hline PAB (g/l) & $180.34 \pm 19.73$ & $191.43 \pm 18.62^{\mathrm{a}, \mathrm{b}}$ & $202.31 \pm 20.04$ & $189.35 \pm 19.82$ & $181.05 \pm 18.47^{\mathrm{a}}$ & $193.10 \pm 19.58$ \\
\hline HGB $(\mathrm{g} / \mathrm{l})$ & $99.67 \pm 4.12$ & $92.32 \pm 4.23^{\mathrm{a}, \mathrm{b}}$ & $97.76 \pm 5.15$ & $99.32 \pm 3.96$ & $80.92 \pm 4.15^{\mathrm{a}}$ & $96.39 \pm 5.02$ \\
\hline $\mathrm{Wt}(\mathrm{kg})$ & $52.21 \pm 9.27$ & $54.13 \pm 7.16^{\mathrm{b}}$ & $52.18 \pm 8.75$ & $52.24 \pm 9.19$ & $50.43 \pm 7.54^{\mathrm{a}}$ & $52.59 \pm 8.71$ \\
\hline
\end{tabular}

${ }^{\mathrm{a}} \mathrm{P}<0.05$ compared with those before operation; ${ }^{\mathrm{b}} \mathrm{P}<0.05$ compared with those in the TPN group. TBL, total bilirubin; ALT, alanine aminotransferase; AST, aspartate transaminase; TP, total protein; PAB, prealbumin; HGB, hemoglobin; Wt, weight.

history (hypertension, coronary heart and disease), family history, operation methods, the operation of the International Federation of Gynecology and Obstetrics (FIGO) in 2012, histological grading, postoperative evacuation time, complications.

Methods. Nitrogen $(0.2 \mathrm{~g} / \mathrm{kg})$ and $125.5 \mathrm{~kJ} /(\mathrm{kg} / \mathrm{day})$ calorie were given to the EEN group and TPN group. The blood glucose of two groups of patients was maintained using the insulin pump, which was monitored once every $6 \mathrm{~h}$, and the pumping quantity was adjusted according to the condition of blood glucose. According to the evacuation condition of patients, the nutrition input was correspondingly decreased. In supplying nutrition, the nutrition was supplied in a slowfast speed, from a small amount to a large amount and from a low concentration to a high concentration, so as to avoid patient discomforts. a) Patients in the EEN group received EEN (nutrition was given through intestinal canals within $24 \mathrm{~h}$ after operation). When the anastomotic stoma of the digestive tract was reconstructed, the nutrition tube was inserted into the jejunum. The nutrition tube was inserted at $30-50 \mathrm{~cm}$ of the flexor ligament in patients receiving Billroth I operation; it was inserted at $30-50 \mathrm{~cm}$ of the distal anastomotic stoma of the gastric jejunum in patients receiving Billroth II operation, and the length of the tube in the jejunum was above $40 \mathrm{~cm}$; it was inserted at $30-50 \mathrm{~cm}$ of the distal anastomotic stoma of the oesophagus jejunum in patients receiving total gastrectomy with one end buried in the tube along the intestinal wall in the tunnel type with the length of approximately $10 \mathrm{~cm}$ and the other end fixed by the parietal peritoneum and skin. At $24 \mathrm{~h}$ after operation, at the first place, patients with gastrointestinal symptoms in the nutrition tube after instilling $300 \mathrm{ml}$ saline were excluded, and those without this symptom further received nutrition support in the jejunum. b) Patients in the TPN group were supported by parenteral nutrition, including amino acids, fat emulsion, carbohydrates, vitamins, electrolytes and other nutrients needed by the body, which were provided by parenteral pathways, and these nutrient solutions were intravenously input after operation.
Observation indexes. Venous blood (3-5 ml) before the elbow was extracted from patients under the fasting state in the early morning, which was measured after the anticoagulation centrifugation and refrigeration. The levels of liver function indexes [total bilirubin (TBL), alanine aminotransferase (ALT) and aspartate transaminase (AST)] and nutritional indexes [total protein (TP), prealbumin (PAB) and hemoglobin (HGB)] were measured before operation and on the 4th day and 8th day after operation. The blood glucose levels of patients at fasting and $2 \mathrm{~h}$ after a meal on the 1st day before operation, the date of operation and the 1st-8th day after operation were dynamically measured.

Statistical analysis. Data were recorded using Statistical Product and Service Solutions (SPSS) 20.0 software (IBM Corp., Armonk, NY, USA). Measurement data were described as [mean \pm standard deviation (SD)] and compared using the t-test and the analysis of variance. Count data were presented as percentage and compared using Fisher's exact test or Chi-square test. $\mathrm{P}<0.05$ was considered to indicate a statistically significant difference.

\section{Results}

Comparison of the improvement of nutritional indexes between the two groups of patients. There were no significant differences in preoperative indexes of patients between the two groups, and the differences were not statistically significant ( $P>0.05)$. On the 8th day after operation, there were no statistically significant differences between the two groups in terms of TBL, ALT, AST, TP, PAB, HGB and weight (Wt) $(\mathrm{P}<0.05)$. On the 4th day after operation, the levels of TBL, ALT, AST, TP, $\mathrm{PAB}, \mathrm{HGB}$ and $\mathrm{Wt}$ in the EEN group were significantly higher than those in the conventional group $(\mathrm{P}<0.05)$ (Table II).

Comparison of postoperative complications, gastrointestinal function recovery and other conditions between the two groups of patients. In the TPN group, there were a total of 29 patients with adverse reactions, including pulmonary infection $(n=5)$, anastomotic fistula $(n=6)$, wound infection $(n=7)$, 
Table III. Comparison of postoperative complications and gastrointestinal function recovery between the two groups of patients.

\begin{tabular}{|c|c|c|c|c|}
\hline Index & EEN group $(n=66)$ & TPN group $(n=63)$ & $\mathrm{t} / \chi^{2}$ value & P-value \\
\hline Pulmonary infection & 0 & $5(7.94)$ & 3.912 & 0.006 \\
\hline Anastomotic fistula & 0 & $6(9.52)$ & 4.235 & 0.004 \\
\hline Wound infection & 0 & $7(11.11)$ & 5.214 & 0.002 \\
\hline Liver function impairment & 0 & $3(4.76)$ & 3.126 & 0.009 \\
\hline Vein catheter infection & 0 & $8(12.70)$ & 5.769 & $<0.001$ \\
\hline Postoperative evacuation time (h) & $38.65 \pm 6.21$ & $53.08 \pm 5.34$ & 2.658 & 0.012 \\
\hline Hospitalization time (days) & $12.3 \pm 4.5$ & $18.1 \pm 3.7$ & 7.253 & $<0.001$ \\
\hline Postoperative hospitalization cost (ten thousand yuan) & $2.4 \pm 1.9$ & $3.3 \pm 0.8$ & 9.561 & $<0.001$ \\
\hline
\end{tabular}

$\mathrm{P}<0.05$ was considered to indicate a statistically significant difference.

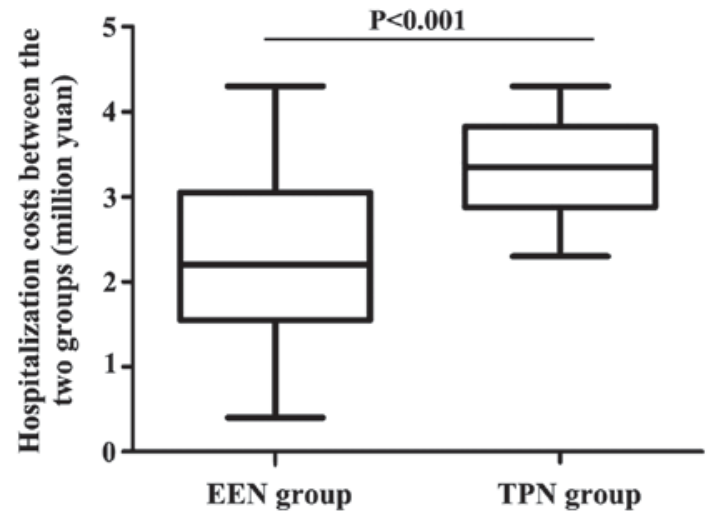

Figure 1. Comparison of hospitalization costs between the two groups of patients. The hospitalization cost in the EEN group is significantly lower than that in the TPN group, and the difference is statistically significant $(\mathrm{P}<0.001)$.

liver function impairment $(n=3)$ and vein catheter infection $(n=8)$; in the EEN group, there was no patient with adverse reactions. The incidence rate of complications in the EEN group was significantly lower than that in the TPN group, and the difference was statistically significant $(\mathrm{P}<0.05)$. The postoperative evacuation time of patients in the TPN group was later than that in the EEN group, and the hospitalization time and cost were higher than those in the EEN group $(\mathrm{P}<0.05)$ (Table III).

Comparison of the hospitalization costs between the two groups of patients. The hospitalization cost in the EEN group was significantly lower than that in the TPN group (2.4 \pm 1.9 vs. $3.3 \pm 0.8)$, and the difference was statistically significant $(\mathrm{P}<0.001)$ (Fig. 1).

Comparison of perioperative blood glucose fluctuation between the two groups of patients. There was no significant difference in preoperative blood glucose between the two groups of patients $(\mathrm{P}>0.05)$, and the preoperative blood glucose in the two groups was comparable. In the comparison of the blood glucose fluctuation level at fasting and $2 \mathrm{~h}$ after a meal, the blood glucose fluctuation values in the TPN group were higher than those in the EEN group, and the differences were statistically significant $\left(\chi^{2}=13.219, P=0.002 ; \chi^{2}=20.527\right.$, $\mathrm{P}<0.001)$, indicating that the blood glucose fluctuation was more obvious in the TPN group, and the blood glucose in the EEN group was stable (Fig. 2).

\section{Discussion}

Patients with malignant tumors often suffer malnutrition, and some of them are manifested as cachexia (5). Poor nutritional status and high blood glucose levels of patients with gastric cancer complicated with diabetes mellitus type 2 are bottlenecks of postoperative recovery. For patients with gastric cancer complicated with diabetes mellitus type 2, surgical trauma stress can increase blood glucose (6), and postoperative fasting intestinal mucosa will shrink, become necrosis and change permeability (7). Nutrition support is divided into enteral nutrition and parenteral nutrition, which is an important means to promote patients' rehabilitation. EEN refers to giving the enteral nutrition support within postoperative 6-24 h (8). Parenteral nutrition is to provide the body with an appropriate amount of three major nutrients, vitamins, electrolytes and water through parenteral pathways (usually through veins), but how to better improve the postoperative nutritional status and control blood glucose level of patients with gastric cancer complicated with diabetes mellitus is the focus and difficulty of the current research.

Studies have shown that after an abdominal operation, the function recovery of the stomach and colon is slow, and small intestine function generally returns to normal within 6-12 h after operation, which is the theoretical basis for EEN (9). EEN is the preferred nutrition support method for patients receiving gastrointestinal operation currently recognized by most scholars (10-12). It is more in line with the physiological state so as to comprehensively provide nutrition with less complications, which is a safe and effective method to effectively improve the visceral function $(13,14)$. It is more convenient to regulate blood glucose by EEN as on the one hand, EEN maintains intestinal mucosal integrity and increases intestinal permeability so as to release glucose-dependent insulinotropic hormones (15), and on the other hand, the addition of dietary fiber in EEN preparations can delay the digestion and absorption rates of carbohydrates (16). This study revealed that in the comparison of the blood glucose fluctuation level of the two groups of patients at fasting and $2 \mathrm{~h}$ after a meal within 8 days after operation, the blood glucose fluctuation values in the 

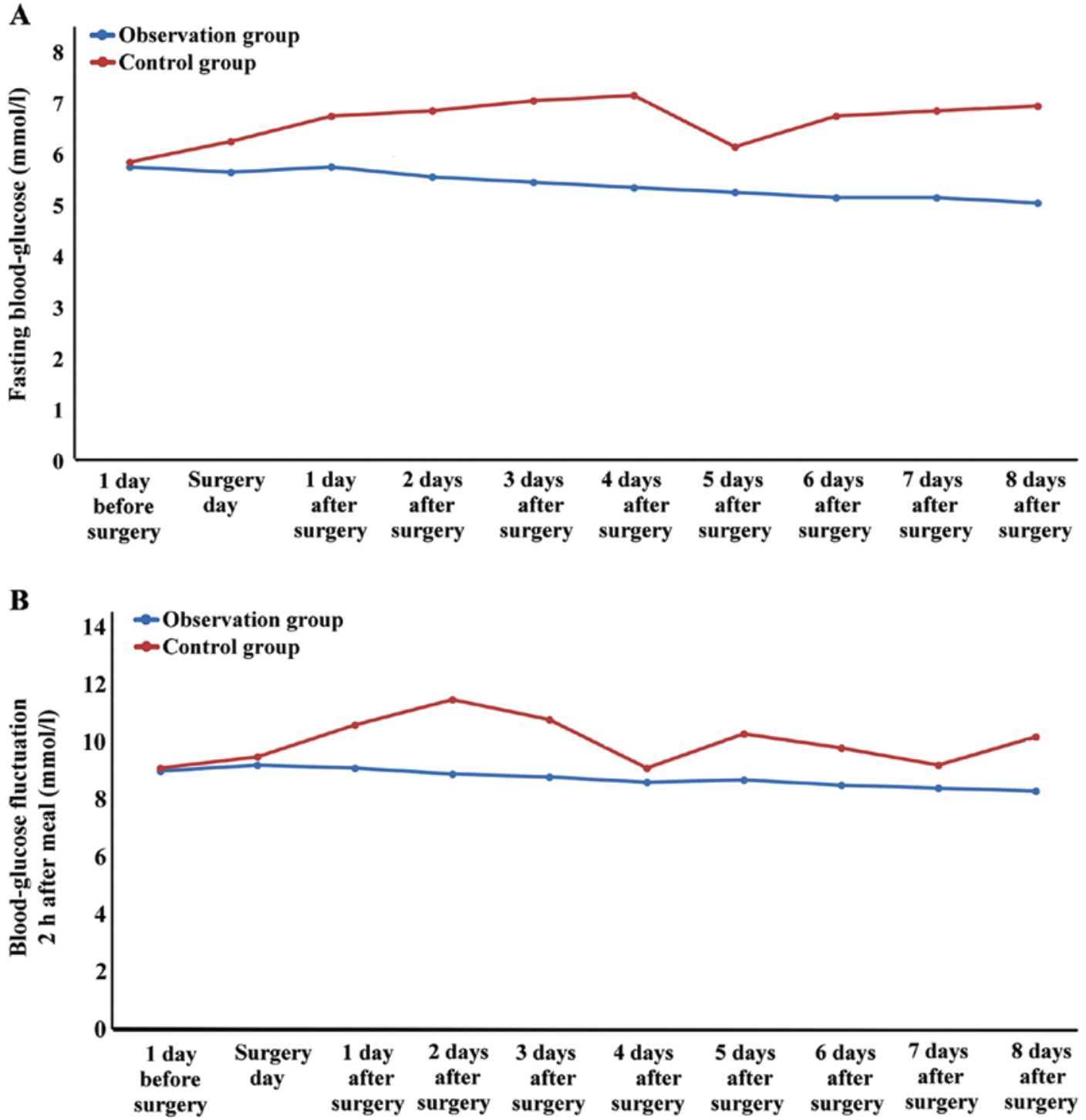

Figure 2. Comparison of blood glucose fluctuation at fasting between the two groups of patients.

TPN group were higher than those in the EEN group $(\mathrm{P}<0.05)$, indicating that it is more convenient to regulate postoperative blood glucose of patients with gastric cancer complicated with diabetes mellitus type 2 by EEN.

EEN is conducive to the growth of intestinal epithelial cells, the prevention of mucosal atrophy and the maintenance of mechanical barrier function. It contributes to the secretion of immunoglobulin A ( $\operatorname{IgA})$ from intestinal cells so as to maintain immunological barriers, avoids the flora shift, which is beneficial to the growth of normal intestinal bacteria and maintains the biological barriers of the intestine. It can also promote the secretion of the stomach (gastrin and stomach acid) to maintain the mucosal chemical barriers, thereby promoting gastrointestinal function recovery. At the same time, EEN stimulates the secretion of digestive juice, hormones and enzymes, promotes gastrointestinal motility and gallbladder contraction and increases visceral blood flow, thus effectively reducing the incidence of hepatobiliary complications (17).

Visceral protein is the most important nutritional monitoring index, including albumin, transferrin, prealbumin and fibronectin. Albumin cannot be the index rapidly reflecting the body's nutritional status as it is easily affected by the intake of protein and energy and liver functions due to its long half-life; transferrin is also rarely applied clinically due to its complex metabolism and susceptibility to many factors; fibronectin with short half-life can only act as a short-term nutrition support index; prealbumin has received much attention in recent years among visceral proteins due to its short half-life, good specificity and the close relation to patient's nutritional status and prognosis (18). Therefore, it is a reliable index to determine the nutritional status of patients, and is one of the indexes selected in this study to determine nutritional status.

Nutritional pathways can cause relevant complications. For example, parenteral nutrition is given mainly by intravenous pathways, whose major complications are catheter-related complications (pneumothorax, peripheral vascular injury, venous thrombosis and air embolism), catheter-induced infections or septicemia, which may even threaten patient lives. There are some problems in the intestinal nutrition clinically, such as discomforts after intubation, nausea, vomiting and diarrhea. However, recent studies have shown that placing a nasogastric tube or a nasointestinal tube for enteral nutrition 
after operation is safe as it does not increase postoperative complications and mortality rates compared with the parenteral nutrition (19).

The incidence of postoperative complications in patients with gastric cancer is affected by different nutritional pathways. A meta-analysis (20) showed that the enteral nutrition can significantly reduce the incidence rate of postoperative complications in patients with gastric cancer, especially in the anastomotic fistula, abdominal abscess and mortality rate, and significantly shorten the hospitalization time. It was also found in this study that there were a total of 29 patients with adverse reactions in the TPN group, but no patient in the EEN group, indicating that the incidence rate of complications in the EEN group was significantly lower than in the TPN group $(\mathrm{P}<0.05)$. EEN group had shorter evacuation time, shorter hospitalization time and less hospitalization cost $(\mathrm{P}<0.05)$, which was consistent with the literature. Different nutrition support pathways affect liver function in different degrees due to the different forms, concentrations and rates of nutrients into the liver. In the enteral nutrition support, the liver function state when patients are eating is not able to be reached, especially for patients receiving long-term TPN as long-term high energy and no foods containing fats passing through the intestine easily cause liver injuries. Liver enzyme abnormalities will occur in $20-40 \%$ of TPN patients 2 weeks after operation or even cholestatic liver dysfunction in severe cases (21).

Different nutrition support pathways exert different effects on glucose metabolism. Generally, it is believed that TPN is more likely to cause metabolic disorders of the body such as glucose metabolism disorder, electrolyte imbalance, acid-base disturbance and azotemia. Among them, the glucose metabolism disorder is the most common one, which may cause hyperosmolar coma for severely ill patients. The reason may be that the intake of glucose per unit time is too high and rapid so as to cause a transient hyperglycemia, while stresses increase gluconeogenesis and the emergence of insulin resistance. If the patient suffers from diabetes mellitus or liver diseases before operation, the applied glucose in vivo is more likely to be limited, thus promoting the increase of blood glucose, and the elevated blood glucose increases the incidence rate of postoperative incision infections (22).

This is a prospective study on patients with gastric cancer complicated with diabetes mellitus type 2 . The EEN pathway was used to provide nutrition with such advantages as less complications, early postoperative evacuation time, short hospitalization time, low cost and stable control of blood glucose. It helps patients with gastric cancer with diabetes mellitus pull through the perioperative period safely, which provides a certain guidance for clinical practice. However, this study is limited to a single-center and small sample size, and it is expected that multi-center study with a large sample size may lead to more meaningful results.

\section{Acknowledgements}

Not applicable.

\section{Funding}

No funding was received.

\section{Availability of data and materials}

The datasets used and/or analyzed during the current study are available from the corresponding author on reasonable request.

\section{Authors' contributions}

JW and JZ collected and analyzed the general data of patients. YZ helped with liver function indexes. JW and CL were responsible for nutritional indexes. All authors have read and approved the final manuscript.

\section{Ethics approval and consent to participate}

This study was approved by the Ethics Committee of The First People's Hospital of Jinan (Jinan, China). Patients were informed of the conditions and signed informed consent.

\section{Consent for publication}

Not applicable.

\section{Competing interests}

The authors declare that they have no competing interests.

\section{References}

1. Smith U and Gale EA: Cancer and diabetes: Are we ready for prime time? Diabetologia 53: 1541-1544, 2010.

2. Bower M, Jones W, Vessels B, Scoggins C and Martin R: Nutritional support with endoluminal stenting during neoadjuvant therapy for esophageal malignancy. Ann Surg Oncol 16: 3161-3168, 2009.

3. Fernández-Sordo JO, Konda VJ, Chennat J, Madrigal-Hoyos E, Posner MC, Ferguson MK and Waxman I: Is endoscopic ultrasound (EUS) necessary in the pre-therapeutic assessment of Barrett's esophagus with early neoplasia? J Gastrointest Oncol 3: 314-321, 2012.

4. American Diabetes Association: Diagnosis and classification of diabetes mellitus. Diabetes Care 30 (Suppl 1): S42-S47, 2007.

5. Makeeva TK and Galkin AA: Nutritional support in the treatment of gastric cancer. Vopr Onkol 55: 237-240, 2009 (In Russian).

6. Finney SJ, Zekveld C, Elia A and Evans TW: Glucose control and mortality in critically ill patients. JAMA 290: 2041-2047, 2003.

7. Yu G, Chen G, Huang B, Shao W and Zeng G: Effect of early enteral nutrition on postoperative nutritional status and immune function in elderly patients with esophageal cancer or cardiac cancer. Chin J Cancer Res 25: 299-305, 2013.

8. Mahmoodzadeh H, Shoar S, Sirati F and Khorgami Z: Early initiation of oral feeding following upper gastrointestinal tumor surgery: A randomized controlled trial. Surg Today 45: 203-208, 2015.

9. Kirby DF and Teran JC: Enteral feeding in critical care, gastrointestinal diseases, and cancer. Gastrointest Endosc Clin N Am 8: 623-643, 1998.

10. Moskovitz DN and Kim YI: Does perioperative immunonutrition reduce postoperative complications in patients with gastrointestinal cancer undergoing operations? Nutr Rev 62: 443-447, 2004.

11. Farreras N, Artigas V, Cardona D, Rius X, Trias M and González JA: Effect of early postoperative enteral immunonutrition on wound healing in patients undergoing surgery for gastric cancer. Clin Nutr 24: 55-65, 2005.

12. Jiang K, Cheng L, Wang JJ, Li JS and Nie J: Fast track clinical pathway implications in esophagogastrectomy. World $\mathrm{J}$ Gastroenterol 15: 496-501, 2009.

13. Zou XP, Chen M, Wei W, Cao J, Chen L and Tian M: Effects of enteral immunonutrition on the maintenance of gut barrier function and immune function in pigs with severe acute pancreatitis. JPEN J Parenter Enteral Nutr 34: 554-566, 2010. 
14. Barlow R, Price P, Reid TD, Hunt S, Clark GW, Havard TJ, Puntis MC and Lewis WG: Prospective multicentre randomised controlled trial of early enteral nutrition for patients undergoing major upper gastrointestinal surgical resection. Clin Nutr 30: 560-566, 2011 .

15. Lidder P, Flanagan D, Fleming S, Russell M, Morgan N Wheatley T, Rahamin J, Shaw S and Lewis S: Combining entera with parenteral nutrition to improve postoperative glucose control. Br J Nutr 103: 1635-1641, 2010.

16. Yang H: Reconsideration on nutritional treatment and nutritional support in patients with diabete mellitus during perioperative period. Chin J Colorec Dis 2: 14-19, 2013.

17. Bower RH, Talamini MA, Sax HC, Hamilton F and Fischer JE: Postoperative enteral vs parenteral nutrition. A randomized controlled trial. Arch Surg 121: 1040-1045, 1986.

18. Chen DW, Wei Fei Z, Zhang YC, Ou JM and Xu J: Role of enteral immunonutrition in patients with gastric carcinoma undergoing major surgery. Asian J Surg 28: 121-124, 2005.
19. Doglietto GB, Papa V, Tortorelli AP, Bossola M, Covino M and Pacelli F; Italian Total Gastrectomy Study Group: Nasojejunal tube placement after total gastrectomy: A multicenter prospective randomized trial. Arch Surg 139: 1309-1313, 2004.

20. Mazaki T and Ebisawa K: Enteral versus parenteral nutrition after gastrointestinal surgery: A systematic review and metaanalysis of randomized controlled trials in the English literature. J Gastrointest Surg 12: 739-755, 2008.

21. Marik PE and Zaloga GP: Early enteral nutrition in acutely ill patients: A systematic review. Crit Care Med 29: 2264-2270, 2001.

22. Hu QG and Zheng QC: The influence of enteral nutrition in postoperative patients with poor liver function. World J Gastroenterol 9: 843-846, 2003.

This work is licensed under a Creative Commons Attribution-NonCommercial-NoDerivatives 4.0 International (CC BY-NC-ND 4.0) License. 